\title{
Development and characterization of Near-UV sensitive Silicon Photomultipliers for the Schwarzschild-Couder Telescope prototype for the Cherenkov Telescope Array
}

\author{
Emanuele FiandriniUniversitá e INFN, Perugia (IT) \\ E-mail: emanuele.fiandriniepg.infn.it \\ G. Ambrosi, M. Ambrosio,C. Aramo,B. Bertucci,E. Bissaldi,A. Boiano, C. \\ Bonavolontà, M. Caprai, N. Giglietto, F. Giordano, M. Ionica, S. Loporchio, M. de \\ Lucia, V. Masone, R. Paoletti, A. Rugliancich, D. Simone, L. Tosti, L. Di Venere, V. \\ Vagelli, M. Valentino for the CTA Consortium
}

\begin{abstract}
Silicon Photomultipliers (SiPM) are standard sensors widely employed for applications in which high sensitivities and fast responses in the detection of low fluxes of visible and UV photons are required. The Italian Institute of Nuclear Physics (INFN), in collaboration with Fondazione Bruno Kessler, is involved in a $R \& D$ project for SiPM sensors sensitive to near UV (NUV) wavelengths. The performances of the latest technology of NUV-High-Density SiPM have confirmed that the quality of the current production technology opens the possibility to employ these devices for many applications. In this contribution, we review the performances of the latest technology of NUV-High-Density SiPM and the prospects for their use in one of the designs of the camera focal planes of Schwarschild-Couder Telescope prototype, including the development of packaging procedures of single sensors into high-density multi-SiPM modules and the development of a custom front-end ASIC for a high rate waveform sampling of the SiPM signals.
\end{abstract}

The European Physical Society Conference on High Energy Physics

5-12 July

Venice, Italy

\footnotetext{
* Speaker.
} 


\section{The CTA Observatory and the SCT telescope proposal}

The Cherenkov Telescope Array (CTA) Consortium aims to build the next generation of Imaging Air Cherenkov Telescope (IACT) arrays for the detection of $\gamma$-rays in the $20 \mathrm{GeV}-300 \mathrm{TeV}$ range [1]. Two arrays of IACTs will be deployed, one in the Southern hemisphere at the Paranal site (Chile) and the other one in the Northern hemisphere on La Palma (Spain) to achieve full sky coverage.

The arrays will cover an area of $\sim 1 \mathrm{~km}^{2}$ in the northern site and a few $\mathrm{km}^{2}$ on southern one with telescopes of different sizes: 4 Large telescopes (LST, $\sim 23$ m diameter), 25 Medium size telescopes (MST, $\sim 12 \mathrm{~m}$ diameter) and 70 Small telescopes (SST, $\sim 4 \mathrm{~m}$ diameter) in the southern site (see caption of Figure 1) to extend the accessible energy range below $50 \mathrm{GeV}$ and up to 300 $\mathrm{TeV}$ and to improve the flux sensitivity by a factor 5-10 in the core energy range with respect the current IACT generation.

This will greatly help to understand the nature of very high energy phenomena, as the origin of cosmic rays, AGN emission, pulsars and to address the nature of dark matter, just to mention a few examples.

This will also allow us to crosscheck between space- and ground-based detectors at low energy and will open a new window on the high energy sky.

CTA is planned to be operated as open observatory accessible to the whole scientific community, with a large amount of data taking time dedicated to observation strategies proposed by the astrophysical community besides the observation time allocated to Key Science Projects (KSPs) managed by the CTA collaboration to address a selection of open key science problems in a coherent strategic approach.

(a)

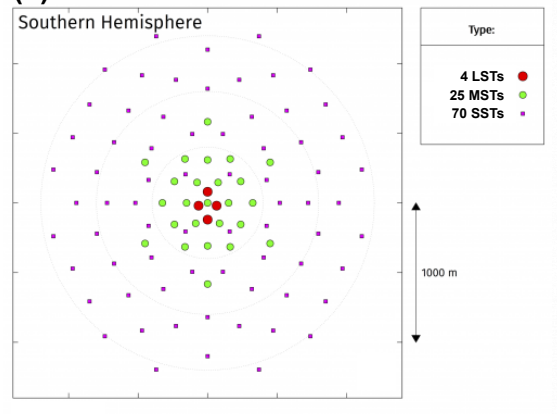

(b)

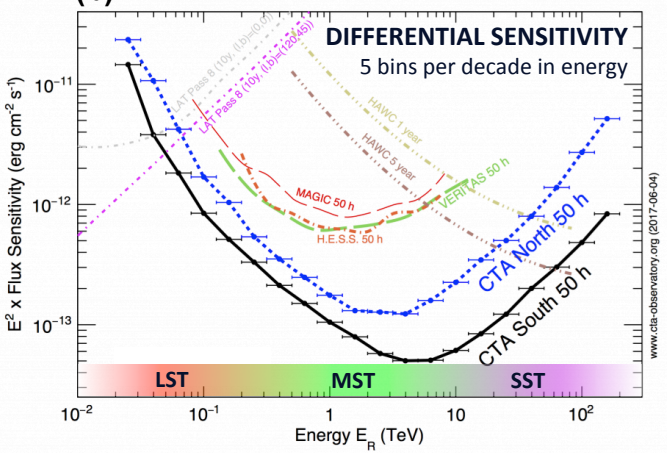

Figure 1: (a) A possible layout of the Southern hemisphere array with 4 Large Size Telescopes (LST, $23 \mathrm{~m}$ mirrors), 25 Medium Size Telescopes (MST, $12 \mathrm{~m}$ mirrors) and 70 Small Size Telescopes (SST, $4 \mathrm{~m}$ mirrors). (b) Expected differential sensitivity for the CTA observatory compared with other ground and space observatories [1].

The possibility of a Schwarzschild-Couder (SC) dual mirror optics design for the CTA Medium Size Telescopes (MST) is currently under investigation for the extension of the CTA array baselines.

In comparison with the single mirror solution, the SC technology minimizes optical aberrations and de-magnifies images, opening the possibility for the use of compact SiPM cameras, 
improving the MST telescope performances at a reduced cost per telescope.

SiPMs offer many advantages with respect to the traditional phototubes: smaller pixel size, hence higher angular resolution, higher Photon Detection Efficiency (PDE) at UV wavelengths, fast response, they can be operated under moon light, their working voltage is below $100 \mathrm{~V}$, and they are light-weight.

A prototype telescope (pSCT) to prove the feasibility of the SC solution for MST telescopes is currently being assembled [2].

\section{A SiPM camera demonstrator for the pSCT telescope}

Near UltraViolet High-Density (NUV-HD) $6 \times 6 \mathrm{~mm}^{2}$ SiPMs with $30 \times 30 \mu \mathrm{m}^{2}$ microcell area, produced by Fondazione Bruno Kessler in collaboration with INFN, are being considered for a possible upgrade of the pSCT camera, currently equipped with Hamamatsu MPPC S12642 modules.

The sensors feature a breakdown voltage of $\sim 28 \mathrm{~V}$ at room temperature with a temperature gradient of $\sim 30 \mathrm{mV} / \mathrm{C}$, a Photo Detection Efficiency (PDE) peak of $\sim 50 \%$ at $350 \mathrm{~nm}$ dropping below $20 \%$ above $500 \mathrm{~nm}$ and a single photon Dark Count rate below $100 \mathrm{kHz} / \mathrm{mm}^{2}$ at $20 \mathrm{C}$.

The performances of the NUV-HD SiPM devices are described in details elsewhere [3].

NUV-HD SiPMs fulfil the performance requirements to equip the cameras of CTA telescopes, and they have been chosen to equip the focal plane camera of the pSCT telescope for a possible replacement of the Hamamatsu MPPCs that are currently used to read the Cherenkov light on the focal plane.

The pSCT camera has a width of $81 \mathrm{~cm}$ for a total field of view of $8^{\circ}$ and will host 11328 $6 \times 6 \mathrm{~mm}^{2}$ sensors grouped in units composed of 64 sensors. Each sensor corresponds to a $0.064^{\circ}$ in the sky, matching the PSF of the optical system. Each $64 \mathrm{SiPM}$ unit covers $54 \times 54 \mathrm{~mm}^{2}$ and is divided in 4 modules composed of 16 pixels.

INFN has designed custom $27 \times 27 \mathrm{~mm}^{2}$ area PCBs with $0.5 \mathrm{~mm}$ sensor-sensor distance to obtain uniform pixel coverage of the modules and of the camera, and compatible with the pSCT camera design (Figure 2).
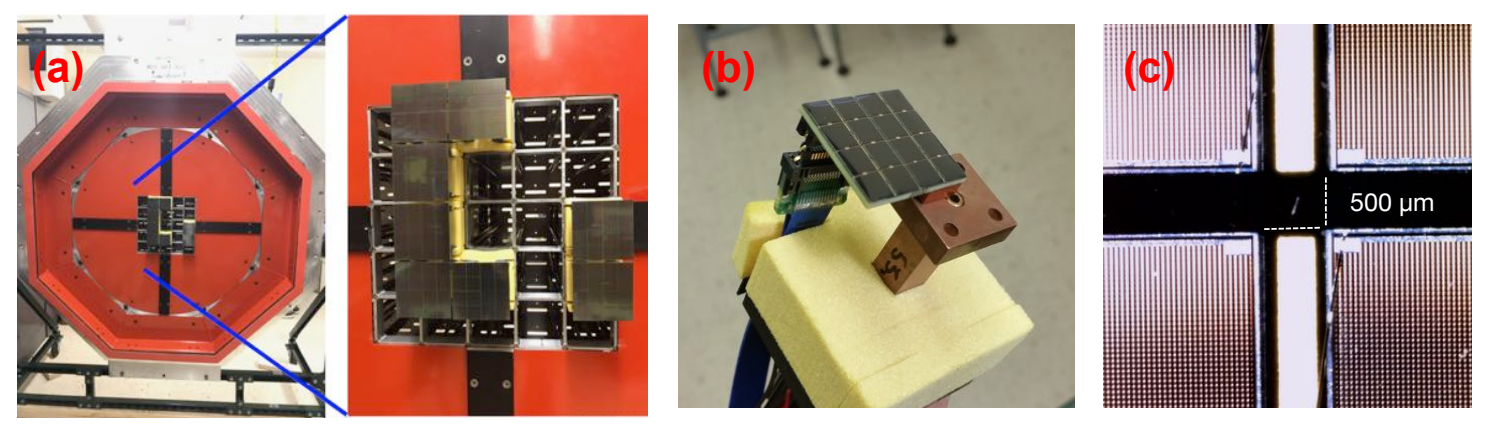

Figure 2: (a): pSCT camera mechanics with 8 quadrants hosting modules with Hamamatsu MPPCs. (b) A 16 NUV-HD SiPM module prototype coupled with the pSCT telescope mechanical unit. (c) Details of one NUV-HD SiPM module, with the SiPM bonding pad wire-bonded to the PCB signal pad for anode readout. 


\section{Packaging and tests of the telescope camera units}

While single sensors are provided by the vendor, the procedures for the assembly and packaging of the modules have been completely developed by INFN.

A manual die-bonder machine is used to dispense the conductive glue on the PCB pads and to precisely place the SiPMs on the PCBs.

The alignment of the sensors, checked with an optical metrology machine, is better than $40 \mu \mathrm{m}$ (Figure 3). The flatness of the modules has been checked using a ruby-head touch probe machine, and the maximum deviation from planarity across the whole modules has been found to be $\sim 80 \mu \mathrm{m}$.

The possibility to use semi-automatic die-bonder machines for future assemblies is under investigation.
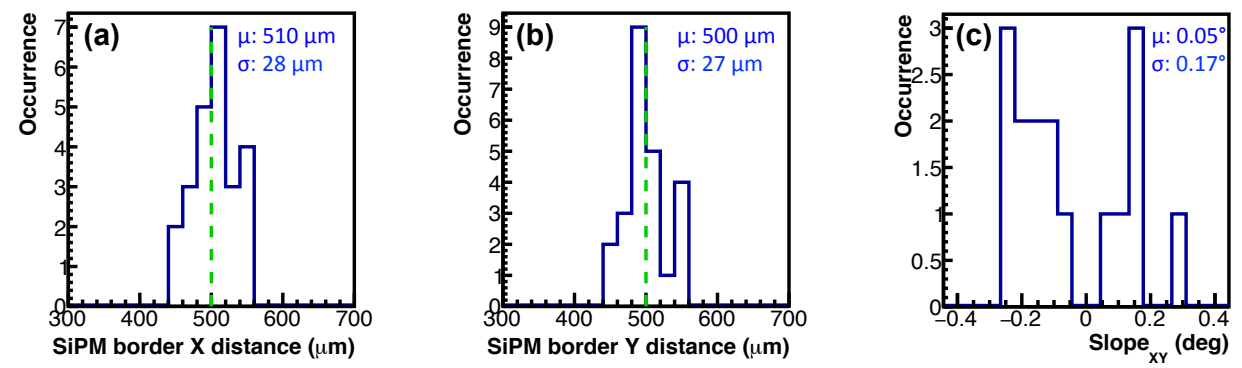

Figure 3: Results of the optical inspection of one prototype module for the distance between sensor edges in the $\mathrm{X}$ (a) and $\mathrm{Y}$ (b) directions and for the sensor rotation in the XY plane (c). Green lines highlight the nominal distances.

Each SiPM anode is bonded to the signal readout pad of the PCB using a $25 \mu \mathrm{m}$ AlSi1\% wire. Subsequently, the breakdown voltage of each sensor is measured analyzing its dark current dependence over the bias voltage to spot any defective sensor to replace.

Fully working modules are finally covered with a UV-transparent epoxy for protection.

The pSCT digitization and readout electronics is based on the TARGET7 ASIC[4]. The readout boards are hosted directly behind the sensor modules inside the camera mechanics.

Each TARGET7 ASIC handles first-level trigger - based on the signal of 4 adjacent sensors - and digitization for 16 channels, sampling the analog signal at $1 \mathrm{GS} / \mathrm{s}$ with a maximum buffer depth of $\sim 16 \mu \mathrm{s}$.

To adapt the current board design to the readout of the prototype modules, the pre-amplification stage of the digitization board has been optimized for the signal of NUV-HD SiPM sensors. Moreover, a mezzanine DC-DC converter has been introduced to optimize the bias voltage for the NUVHD sensors.

Currently the performances of the modules coupled with the modified readout electronics are being validated. Signal waveforms have been recorded illuminating the devices with low intensity $380 \mathrm{~nm}$ pulsed laser for different operating voltages (Figure 4(a)).

Preliminary results show a net separation between photoelectron peaks in the integrated charge distribution (Figure 4(b)), a good gain linearity checked up to $\mathrm{O}(10)$ photoelectrons and a signal to noise ratio for the first photoelectron peak better than 3 and improving for higher charges. 


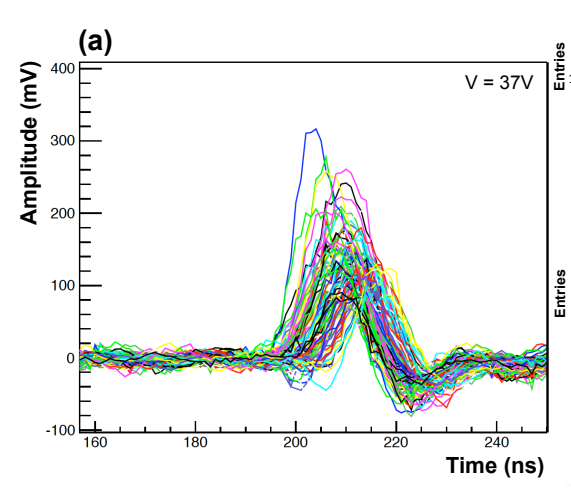

(b)

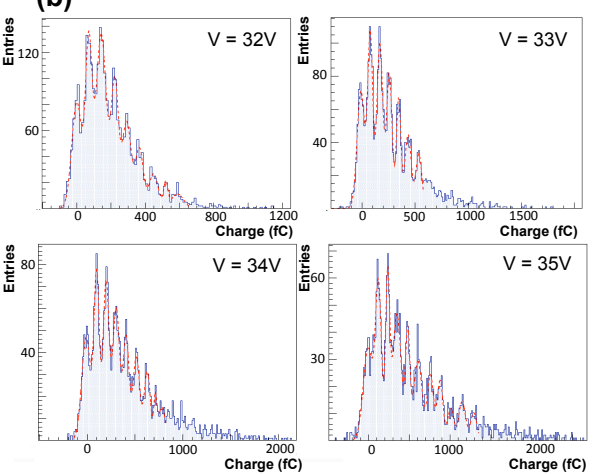

Figure 4: (a) Waveforms recorded with the TARGET7 readout board. (b) Charge spectrum of the signals for different operation voltages.

\section{Outlook and Prospects}

FBK NUV-HD SiPM technology has been tested and its performances are compatible with the requirements to equip the focal planes of CTA telescopes.

Multi-SiPM modules have been developed to equip a possible upgrade of the Medium Size Schwarzschild-Couder telescope prototype pSCT.

36 modules are planned to be installed on the pSCT camera for validation in situ by the end of 2017, to prepare for the next mass production of modules to possibly equip a complete sector of 25 quadrants of pSCT (100 modules composed of 16 SiPMs).

The modules recently assembled are currently tested for validation of their performances, while more recent technologies of SiPMs with similar size are being studied for the next mass production of modules.

\section{Aknowledgements}

The authors acknowledge the support from Progetto Premiale TECHE.it.

\section{References}

[1] B. Acharya et al, Astrop. Phys. 43 (2013) 3; www.cta-observatory.org, 2017/06/12

[2] W. Benbow et al. arXiv:1610.03865 [astro-ph.IM] (2016).

[3] G. Ambrosi et al, IL NUOVO CIMENTO 40 C (2017) 78; A. Otte et al in Nucl. Instrum. Meth. A846 (2017) 106-125;

[4] L. Tibaldo et al, arXiv:1508.06296 [astro-ph.IM] (2015). 